\title{
Azimuthal electric field in a static rotationally symmetric $(2+1)$-dimensional spacetime
}

\author{
Mauricio Cataldo \\ Departamento de Física, Facultad de Ciencias, \\ Universidad del Bío-Bí, Avenida Collao 1202, \\ Casilla 5-C, Concepción, Chile.
}

(Dated: November 13, 2018)

\begin{abstract}
The fundamental metrics, which describe any static three-dimensional Einstein-Maxwell spacetime (depending only on a unique spacelike coordinate), are found. In this case there are only three independent components of the electromagnetic field: two for the vector electric field and one for the scalar magnetic field. It is shown that we can not have any superposition of these components of the electric and magnetic fields in this kind of static gravitational field. One of the electrostatic Einstein-Maxwell solutions is related to the magnetostatic solution by a duality mapping, while the second electrostatic gravitational field must be solved separately. Solutions induced by the more general (2+1)-Maxwell tensor on the static cylindrically symmetric spacetimes are studied and it is shown that all of them are also connected by duality mappings.
\end{abstract}

PACS numbers: 04.20.Jb

In (3+1)-gravity coupled to Maxwell electrodynamics, the Reissner-Nordström black hole solution describes a vacuum static spherically symmetric spacetime endowed with electric $q_{e}$ or magnetic $q_{m}$ charge. If one includes the cosmological constant $\Lambda$, the Reissner-NordströmKottler solution may be written as [1]

$$
d s^{2}=-f(r) d t^{2}+\frac{d r^{2}}{f(r)}+r^{2}\left(d \theta^{2}+\sin ^{2} \theta d \phi^{2}\right)
$$

where

$$
f(r)=1-\frac{2 M G}{r}+\frac{4 \pi G\left(q_{e}^{2}+q_{m}^{2}\right)}{r^{2}}-\frac{1}{3} \Lambda r^{2} .
$$

Here, the solution has radial electric and magnetic fields given by $E(r)=q_{e} / r^{2}$ and $B(r)=q_{m} / r^{2}$ respectively. The case $q_{m}=0$ describes a rest charge at the origin of spherical coordinates and $q_{e}=0$ may be interpreted as a magnetic monopole. The magnetically charged Reissner-Nordström solution is connected to the electrically charged solution, since all the cases with an electric field without sources can be reformulated to be the corresponding cases with a magnetic field (dual to the initial electric one) or mixtures of both fields (through a duality rotation). In all these cases the form of the metric is the same.

The Einstein-Maxwell theories in (2+1)-dimensions without cosmological constant have been discussed by several authors [2, 3, 代. In [4] the authors found the first three-dimensional static electrically charged space-time. Later the inclusion of the cosmological constant was considered. In (2+1)-dimensions there exist the electric and magnetic analogs to the Reissner-Nordström-Kottler solution. They are the static Bañados-Teitelboim-Zanelli

\footnotetext{
*mcataldo@ubiobio.cl
}

(BTZ) black hole $\left(E=q_{e} / r\right)[5]$

$$
\begin{aligned}
d s^{2}= & \left(r^{2} / l^{2}-q_{e}^{2} \ln r-M\right) d t^{2} \\
& -\frac{d r^{2}}{\left(r^{2} / l^{2}-q_{e}^{2} \ln r-M\right)}-r^{2} d \theta^{2},
\end{aligned}
$$

and the Hirschmann-Welch (HW) magnetic solution $\left(B=q_{m} / r\right)$ [6, ]

$$
d s^{2}=\left(r^{2} / l^{2}-M\right) d t^{2}-\frac{r^{2} d r^{2}}{\tilde{g}(r)\left(r^{2} / l^{2}-M\right)}-\tilde{g}(r) d \theta^{2},
$$

where $\tilde{g}(r)=r^{2}+q_{m}^{2} \ln \left(r^{2} / l^{2}-M\right), \Lambda=-1 / l^{2}<0, q_{e}$ is the electric charge, $q_{m}$ magnetic charge and $M$ is the mass of the BTZ black hole. Both solutions are asymptotically anti-de Sitter, like the Reissner-NordströmKottler solution for $\Lambda<0$. In Ref. [7] it is shown that the both solutions (3) and (4) are connected by a duality transformation. Rotating (2+1)-Einstein-Maxwell solutions have been obtained by several authors using an appropriate coordinate transformation [8, 9. Others solutions also have been obtained assuming selfdual (or anti-self-dual) condition imposed on the orthonormal basis components of the electric and magnetic fields 10, 11, 12]. Note that the presence of divergences at spatial infinity in the mass and angular momentum is an usual feature in electrically charged solutions [13]. The authors of the Ref. 111 and 114, 15 regularize these divergences by taking into account a boundary contribution or by the introduction of a topological ChernSimons term respectively. We note also that the magnetic solution (4) has been extended in order to include (i) angular momentum, (ii) the definition of conserved quantities, (iii) upper bounds for the conserved quantities themselves, and (iv) a new interpretation for the magnetic field source [16].

In this letter we shall show that the above description for he static case is incomplete, and then we will focus our 
attention on a complete description for all electrostatic and magnetostatic spacetimes in $(2+1)$-dimensions and on the Einstein-Maxwell theory for cylindrically static spacetimes.

In $(3+1)$-dimensions the Maxwell tensor and its dual are two-forms, but in (2+1)-dimensions the Maxwell tensor is a two-form and its dual is a one-form ${ }^{*} F_{a}=$ $1 / 2 \epsilon_{a b c} F^{b c}(a, b, c=0,1,2)$. This means that the duality transformation which takes place for the ReissnerNordström-Kottler black hole does not take place for $(2+1)$-dimensions.

Now we shall study the three-dimensional EinsteinMaxwell theory for any static spacetime which depends only on a unique spacelike coordinate. The fundamental metric which describes any such static $(2+1)$-dimensional spacetime manifold may be written (in the coordinate system which employs the Killing parameter, $t$, as one of the coordinates) as 17

$$
d s^{2}=e^{2 \alpha\left(x^{1}\right)} d t^{2}-\sum_{a, b=1}^{2} h_{a b}\left(x^{1}\right) d x^{a} d x^{b},
$$

where $e^{2 \alpha\left(x^{1}\right)}=\xi^{c} \xi_{c}(c=0,1,2)$, being $\xi^{c}$ a timelike Killing vector field. By an appropriate change of coordinate the orthogonal part to $\xi^{c}$ may be converted into a diagonal form (see for example [18]) and then the metric (5) may be written in the following form $\left(t=x^{0}\right)$

$$
d s^{2}=e^{2 \alpha}\left(d x^{0}\right)^{2}-e^{2 \beta}\left(d x^{1}\right)^{2}-e^{2 \gamma}\left(d x^{2}\right)^{2},
$$

where $\alpha, \beta$ and $\gamma$ are all functions of the spacelike coordinate $x^{1}$.

In $(2+1)$-dimensions the Maxwell tensor has the form

$$
\begin{array}{r}
F=-E_{1} d x^{0} \wedge d x^{1}-E_{2} d x^{0} \wedge d x^{2} \\
+B d x^{1} \wedge d x^{2},
\end{array}
$$

i.e. the Maxwell tensor has only three independent components: two for the vector electric field and one for the scalar magnetic field. by

We now introduce an orthonormal 1-form basis given

$$
\theta^{(0)}=e^{\alpha\left(x^{1}\right)} d x^{0}, \theta^{(1)}=e^{\beta\left(x^{1}\right)} d x^{1}, \theta^{(2)}=e^{\gamma\left(x^{1}\right)} d x^{2} .
$$

The components of the electric and magnetic field measured by a locally lorentzian observer in this orthonormal basis are $E_{(0)}, E_{(1)}$ and $B_{()}$. Then, the $(2+1)$-Maxwell tensor (7) may be written as

$$
\begin{aligned}
F=E_{(1)}\left(x^{1}\right) \theta^{(1)} \wedge \theta^{(0)} & +E_{(2)}\left(x^{1}\right) \theta^{(2)} \wedge \theta^{(0)} \\
& +B_{()}\left(x^{1}\right) \theta^{(1)} \wedge \theta^{(2)} .
\end{aligned}
$$

The stress tensor of the electromagnetic field we shall take in the same form as in $(3+1)$-dimensions, i.e.

$$
\begin{gathered}
T_{(a)(b)} \theta^{(a)} \otimes \theta^{(b)}=-\frac{1}{4 \pi}\left[F_{(a)(c)} F_{(b)}{ }^{(c)}\right. \\
\left.-\frac{1}{4} g_{(a)(b)} F_{(c)(d)} F^{(c)(d)}\right] \theta^{(a)} \otimes \theta^{(b)}
\end{gathered}
$$

since there is an ambiguity in the definition of the gravitational constant $G$ (there is no Newtonian gravitational limit in (2+1)-dimensions).

In the basis (8) the components of the electromagnetic energy-momentum tensor are

$$
\begin{array}{r}
T^{2+1}=-\frac{1}{8 \pi}\left[-\left(E_{(1)}^{2}+E_{(2)}^{2}+B_{()}^{2}\right) \theta^{(0)} \otimes \theta^{(0)}\right. \\
+\left(E_{(1)}^{2}-E_{(2)}^{2}-B_{()}^{2}\right) \theta^{(1)} \otimes \theta^{(1)} \\
-\left(E_{(1)}^{2}-E_{(2)}^{2}+B_{()}^{2}\right) \theta^{(2)} \otimes \theta^{(2)} \\
+2 E_{(2)} B_{()}\left(\theta^{(0)} \otimes \theta^{(1)}+\theta^{(1)} \otimes \theta^{(0)}\right) \\
-2 E_{(1)} B_{()}\left(\theta^{(0)} \otimes \theta^{(2)}+\theta^{(2)} \otimes \theta^{(0)}\right) \\
\left.+2 E_{(1)} E_{(2)}\left(\theta^{(1)} \otimes \theta^{(2)}+\theta^{(2)} \otimes \theta^{(1)}\right)\right] .
\end{array}
$$

Then we have the following non-trivial components for Einstein's equations with the cosmological constant:

$$
\begin{gathered}
e^{-2 \beta}\left(\gamma^{\prime \prime}+\gamma^{\prime 2}-\gamma^{\prime} \beta^{\prime}\right)= \\
-\Lambda-\frac{\kappa}{8 \pi}\left(E_{(1)}^{2}+E_{(2)}^{2}+B_{()}^{2}\right) \\
-\alpha^{\prime} \gamma^{\prime} e^{-2 \beta}=\Lambda+\frac{\kappa}{8 \pi}\left(E_{(1)}^{2}-E_{(2)}^{2}-B_{()}^{2}\right), \\
e^{-2 \beta}\left(\alpha^{\prime} \beta^{\prime}-\alpha^{\prime \prime}-\alpha^{\prime 2}\right)= \\
\Lambda-\frac{\kappa}{8 \pi}\left(E_{(1)}^{2}-E_{(2)}^{2}+B_{()}^{2}\right) \\
E_{(2)} B_{()}=0 \\
E_{(1)} B_{()}=0
\end{gathered}
$$

and

$$
E_{(1)} E_{(2)}=0
$$

where the prime denotes the differentiation $d / d r$.

Now we must consider the Maxwell equations. The contravariant density components of (7) are

$$
\begin{aligned}
\sqrt{-g} F^{a b}=-2 E_{(1)} e^{\gamma} \delta_{x^{0}}^{[a} \delta_{x^{1}}^{b]} & -2 E_{(2)} e^{\beta} \delta_{x^{0}}^{[a} \delta_{x^{2}}^{b]} \\
& +2 B_{()} e^{\alpha} \delta_{x^{1}}^{[a} \delta_{x^{2}}^{b]}
\end{aligned}
$$

Then, from the source-free Maxwell equations $\left(\sqrt{-g} F^{a b}\right)_{, b}=0$ we obtain the constraints

$$
E_{(1)}=C_{1} e^{-\gamma}, B_{()}=C_{2} e^{-\alpha},
$$

and $E_{(2)}$ remains to be an arbitrary function of the $x^{1}$ coordinate (here $C_{1}$ and $C_{2}$ are constants of integration).

Although for these $(2+1)$-dimensional solutions no duality rotation exists, there is a duality mapping which connects the electric field $E_{(1)}$ and the scalar magnetic field $B_{()}$. Effectively, from (12)-(17) we see that the 
Einstein-Maxwell equations are invariant under the duality mapping

$$
\begin{array}{r}
\alpha \longrightarrow \gamma, \gamma \longrightarrow \alpha \\
E_{(1)} \longrightarrow i B_{()}, B_{()} \longrightarrow i E_{(1)} .
\end{array}
$$

Notice that from the Einstein-Maxwell equations we conclude that either $E_{(1)}$, or $E_{(2)}$ or the magnetic field $B_{()}$ must be zero, i.e. we can not have any superposition of these fields in any three-dimensional static spacetime.

The solution for $E_{(2)}=B_{()}=0$ may be written in the form (6), where

$$
e^{2 \alpha}=e^{-2 \beta}=C-\Lambda\left(x^{1}\right)^{2}-q_{e}^{2} \ln \left(x^{1}\right), \quad e^{\gamma}=x^{1},
$$

$E_{(1)}=q_{e} / x^{1}$ and $C$ is a constant of integration. The solution for $E_{(1)}=E_{(2)}=0$ may be obtained by using on (6) and on (21) the duality mapping (20) in the form [7]

$$
x^{0} \longrightarrow i x^{2}, x^{2} \longrightarrow i x^{0}, q_{e} \longrightarrow i q_{m} .
$$

From these equations we conclude that we have a twodimensional duality in the plane $x^{0} / x^{2}$.

Then, we have that the magnetostatic solution has the form (6), where

$$
e^{\alpha}=x^{1}, e^{-2 \beta}=e^{2 \gamma}=C-\Lambda\left(x^{1}\right)^{2}+q_{m}^{2} \ln \left(x^{1}\right)
$$

and $B_{()}=q_{m} / x^{1}$.

The case for $E_{(1)}=B_{()}=0$ completes the solution of the $(2+1)$-static Einstein-Maxwell problem. It is interesting to note that the Maxwell equations do not fix the form of the $E_{(2)}$ component of the electric field and that this is done by the Einstein equations. This situation also takes place for the magnetic field in the ReissnerNordström solution in $(3+1)$-dimensions.

We now shall find the solution for any static $(2+1)$ dimensional spacetime, and for the electric field component which is not related to the scalar magnetic field by a duality mapping. From (12) and (13) we find

$$
\left(\gamma^{\prime} e^{\alpha-\beta+\gamma}\right)^{\prime}=-2 \Lambda e^{\alpha+\beta+\gamma}
$$

and from (13) and (14) we find

$$
\left(\alpha^{\prime} e^{\alpha-\beta+\gamma}\right)^{\prime}=-2 \Lambda e^{\alpha+\beta+\gamma}
$$

This system can be solved exactly if we suppose, without any loss of generality, that $e^{\beta}=1$. Then we have that

$$
\left(\left(\alpha^{\prime}+\gamma^{\prime}\right) e^{\alpha+\gamma}\right)^{\prime}=-4 \Lambda e^{\alpha+\gamma},
$$

from which we obtain the following solutions:

- For $\Lambda=0$ we find

$$
e^{\alpha}=\left(C x^{1}+D\right)^{(1-F)}, e^{\gamma}=\left(C x^{1}+D\right)^{F},
$$

where $C, D$ and $F$ are integration constants. Re-scaling the $x^{1}$-coordinate we finally find

$$
d s^{2}=\left(x^{1}\right)^{2 F}\left(d x^{0}\right)^{2}-\left(x^{1}\right)^{2 F}\left(d x^{1}\right)^{2}-\left(x^{1}\right)^{2}\left(d x^{2}\right)^{2},
$$

and

$$
E_{(2)}^{2}=\frac{8 \pi F}{\kappa}\left(x^{1}\right)^{-2(F+1)}
$$

from here we see that $F \geq 0$.

- For $\Lambda=-1 / l^{2}<0$ we find

$$
\begin{gathered}
e^{2 \alpha}=e^{-2 \tilde{G} I^{-}}\left(C \sinh \left(2 x^{1} / l\right)+D \cosh \left(2 x^{1} / l\right)\right), \\
e^{2 \gamma}=e^{2 \tilde{G} I^{-}}\left(C \sinh \left(2 x^{1} / l\right)+D \cosh \left(2 x^{1} / l\right)\right),
\end{gathered}
$$

where

$$
I^{-}=\frac{l}{\sqrt{D^{2}-C^{2}}} \arctan \left(\frac{D \tanh \left(x^{1} / l\right)+C}{\sqrt{D^{2}-C^{2}}}\right) .
$$

- For $\Lambda=1 / l^{2}>0$ we find

$$
\begin{gathered}
e^{2 \alpha}=e^{-2 \tilde{G} I^{+}}\left(C \sin \left(2 x^{1} / l\right)+D \cos \left(2 x^{1} / l\right)\right), \\
e^{2 \gamma}=e^{2 \tilde{G} I^{+}}\left(C \sin \left(2 x^{1} / l\right)+D \cos \left(2 x^{1} / l\right)\right),
\end{gathered}
$$

where now

$$
I^{+}=\frac{l}{\sqrt{D^{2}+C^{2}}} \arctan \left(\frac{D \tanh \left(x^{1} / l\right)-C}{\sqrt{D^{2}+C^{2}}}\right) .
$$

Here $\tilde{G}$ is a constant of integration and we have introduced the parameter $l$ related to $\Lambda$ for further use.

From now on we shall assume the spacetime to be statically and rotationally symmetric, implying the existence of a timelike Killing vector $\eta=\partial_{t}$ and a spacelike Killing vector $\zeta=\partial_{\varphi}$. This allows us to identify $x^{0}=t, x^{1}=r$ (the radial coordinate) and $x^{2}=\varphi$ where $-\infty<t<\infty$, $0<r<\infty$ and $0<\varphi<2 \pi$. In this case $E_{r}(r)$ is the radial electric field, $E_{\varphi}(r)$ is the azimuthal electric field and $B(r)$ is the scalar magnetic field.

The rotationally symmetric spacetimes may be classified as follows: For the radial electric field we have the BTZ solution (3), which is obtained from (21) by identifying $C=-M$ and $\Lambda=-1 / l^{2}$. For the scalar magnetic field we have the HW solution (14), which is obtained from (23) by making the same identifications for $C$ and $\Lambda$, and re-scaling the $r$-coordinate.

The case for the azimuthal electric field has not been studied up to now. For this case we shall consider a particular solution given by $\tilde{G}=0$. In this case the obtained solutions take a simple form. Effectively, making $\rho^{2}=C \sinh (2 r / l)+D \cosh (2 r / l)$ and $\rho^{2}=C \sin (2 r / l)+$ $D \cos (2 r / l)$ we have

$$
d s^{2}=\rho^{2} d t^{2}-\frac{d \rho^{2}}{q^{2} / \rho^{2} \pm \rho^{2} / l^{2}}-\rho^{2} d \varphi^{2},
$$

for $\Lambda=-1 / l^{2}$ (the plus sign) and $\Lambda=1 / l^{2}$ (the minus sign) respectively. In this case the azimuthal component 
of the electric field is given by $E_{(2)}=\frac{q}{\sqrt{G} r^{2}}$. If $\Lambda=0$, we have a particular metric of $(28)$ specified by $F=1$. On the other hand, if $q=0$ and $\Lambda=-1 / l^{2}<0$, the vacuum state is obtained, namely, what is to be regarded as empty anti-de Sitter space.

It is interesting to note that these solutions are related to a subset of solutions to the gravitating $O(3) \sigma$ model in $(2+1)$-dimensions [19].

Let us now study the kind of solutions induced by (9) (with $B_{()}=: B_{(3)}$ ) on the time-independent cylindrically symmetric spacetimes when the Einstein-Maxwell theory is considered. Any cylindrically static spacetime admits the Killing vectors $\eta=\partial_{t}, \zeta=\partial_{\varphi}, \xi=\partial_{z}$ and the metric can be written in the form

$$
d s^{2}=e^{2 \alpha(r)} d t^{2}-e^{2 \beta(r)} d r^{2}-e^{2 \gamma(r)} d \varphi^{2}-e^{2 \delta(r)} d z^{2}
$$

We now introduce an orthonormal 1-form basis given by

$$
\begin{aligned}
& \theta^{(0)}=e^{\alpha(r)} d t, \quad \theta^{(1)}=e^{\beta(r)} d r \\
& \theta^{(2)}=e^{\gamma(r)} d \varphi, \quad \theta^{(3)}=e^{\delta(r)} d z .
\end{aligned}
$$

In this case we shall take the Maxwell tensor in the form

$$
\begin{aligned}
F=-E_{(1)} \theta^{(0)} & \wedge \theta^{(1)}-E_{(2)} \theta^{(0)} \wedge \theta^{(2)} \\
& +B_{(3)} \theta^{(1)} \wedge \theta^{(2)}
\end{aligned}
$$

Now $E_{(1)}$ is a radial electric field, $E_{(2)}$ is an azimuthal electric field and $B_{(3)}$ is a magnetic field along the $\mathrm{z}$ direction (we can include here for example an electric component $E_{(3)}$ pointing along the $z$ direction but this component is related by a duality rotation to $\left.B_{(3)}\right)$. These components of the electric and magnetic field are measured by a locally lorentzian observer in the orthonormal basis (38). From (10) we obtain for (39)

$$
\begin{aligned}
T^{3+1}=-\frac{1}{8 \pi}[ & -\left(E_{(1)}^{2}+E_{(2)}^{2}+B_{(3)}^{2}\right) \theta^{(0)} \otimes \theta^{(0)} \\
& +\left(E_{(1)}^{2}-E_{(2)}^{2}-B_{(3)}^{2}\right) \theta^{(1)} \otimes \theta^{(1)} \\
& -\left(E_{(1)}^{2}-E_{(2)}^{2}+B_{(3)}^{2}\right) \theta^{(2)} \otimes \theta^{(2)} \\
& +\left(B_{(3)}^{2}-E_{(1)}^{2}-E_{(2)}^{2}\right) \theta^{(3)} \otimes \theta^{(3)} \\
+ & E_{(2)} B_{(3)}\left(\theta^{(0)} \otimes \theta^{(1)}+\theta^{(1)} \otimes \theta^{(0)}\right) \\
- & 2 E_{(1)} B_{(3)}\left(\theta^{(0)} \otimes \theta^{(2)}+\theta^{(2)} \otimes \theta^{(0)}\right) \\
+ & \left.2 E_{(1)} E_{(2)}\left(\theta^{(1)} \otimes \theta^{(2)}+\theta^{(2)} \otimes \theta^{(1)}\right)\right] .
\end{aligned}
$$

The Einstein equations with cosmological constant are

$$
\begin{gathered}
e^{-2 \beta}\left(-\gamma^{\prime \prime}-\gamma^{\prime 2}+\beta^{\prime} \gamma^{\prime}-\delta^{\prime \prime}-\delta^{\prime 2}+\beta^{\prime} \delta^{\prime}\right. \\
\left.-\gamma^{\prime} \delta^{\prime}\right)=\frac{\kappa}{8 \pi}\left(E_{(1)}^{2}+E_{(2)}^{2}+B_{(3)}^{2}\right)+\Lambda \\
e^{-2 \beta}\left(-\alpha^{\prime} \gamma^{\prime}-\alpha^{\prime} \delta^{\prime}-\gamma^{\prime} \delta^{\prime}\right) \\
=\frac{\kappa}{8 \pi}\left(E_{(1)}^{2}-E_{(2)}^{2}-B_{(3)}^{2}\right)+\Lambda
\end{gathered}
$$

$$
\begin{array}{r}
e^{-2 \beta}\left(-\alpha^{\prime \prime}-\alpha^{\prime 2}+\alpha^{\prime} \beta^{\prime}-\delta^{\prime \prime}-\delta^{\prime 2}-\alpha^{\prime} \delta^{\prime}\right. \\
\left.+\beta^{\prime} \delta^{\prime}\right)=-\frac{\kappa}{8 \pi}\left(E_{(1)}^{2}-E_{(2)}^{2}+B_{(3)}^{2}\right)+\Lambda, \\
e^{-2 \beta}\left(-\alpha^{\prime \prime}-\alpha^{\prime 2}+\alpha^{\prime} \beta^{\prime}-\gamma^{\prime \prime}-\gamma^{\prime 2}-\alpha^{\prime} \gamma^{\prime}\right. \\
\left.+\beta^{\prime} \gamma^{\prime}\right)=\frac{\kappa}{8 \pi}\left(B_{(3)}^{2}-E_{(1)}^{2}-E_{(2)}^{2}\right)+\Lambda,
\end{array}
$$

and

$$
E_{(2)} B_{(3)}=0, E_{(1)} B_{(3)}=0, E_{(1)} E_{(2)}=0,
$$

where the prime denotes the differentiation $d / d r$. The source-free Maxwell equations are now

$$
E_{(1)}=\tilde{C}_{1} e^{-\gamma-\delta}, B_{(3)}=\tilde{C}_{2} e^{-\alpha-\delta},
$$

and $E_{(2)}$ remains to be an arbitrary function of the rcoordinate (here $\tilde{C}_{1}$ and $\tilde{C}_{2}$ are constants of integration).

From eqs. (41)-(46) we see that, under the following duality mappings, the Einstein-Maxwell equations are invariant:

$$
\begin{gathered}
\alpha \longrightarrow \gamma, \gamma \longrightarrow \alpha, E_{(1)} \longrightarrow i B_{(3)}, B_{(3)} \longrightarrow i E_{(1)}, \\
\alpha \longrightarrow \delta, \delta \longrightarrow \alpha, E_{(1)} \longrightarrow i E_{(2)}, E_{(2)} \longrightarrow i E_{(1)}, \\
\gamma \longrightarrow \delta, \delta \longrightarrow \gamma, E_{(2)} \longrightarrow B_{(3)}, B_{(3)} \longrightarrow E_{(2)}
\end{gathered}
$$

We conclude from the cylindrical Einstein-Maxwell equations that we can not consider simultaneously all components for the electromagnetic field given by (39), and thus we have that either the radial, or the azimuthal electric field, or the magnetic field must be zero.

Thus all the considered solutions may be obtained from just one given solution. Witten [20] obtained the solution for a magnetic field along the $z$-axis for $\Lambda=0$. In this case the metric has the form

$$
d s^{2}=r^{2 m^{2}} G^{2}\left(d t^{2}-d r^{2}\right)-G^{-2} d \varphi^{2}-r^{2} G^{2} d z^{2},
$$

where $G=A r^{m}+B r^{-m}$ with $A, B$ and $m$ constants. We shall consider in detail one particular solution for this metric: the well known Melvin-Bonnor universe, which is given by 21

$$
\begin{array}{r}
d s^{2}=\left(1+\frac{b^{2}}{4} r^{2}\right)^{2}\left(d t^{2}-d r^{2}-d z^{2}\right) \\
-r^{2}\left(1+\frac{b^{2}}{4} r^{2}\right)^{-2} d \varphi^{2}
\end{array}
$$

and $B_{(3)}=(b / \sqrt{G})\left(1+\frac{b^{2}}{4} r^{2}\right)^{-2}$. This metric may be generalized to the case where $\Lambda \neq 0$. In another gauge it takes the form

$$
\begin{gathered}
d s^{2}=r^{2}\left(d t^{2}-d z^{2}\right)-\left(\frac{C}{r}-\frac{q_{m}^{2}}{r^{2}}-\frac{\Lambda}{3} r^{2}\right)^{-1} d r^{2} \\
-\left(\frac{C}{r}-\frac{q_{m}^{2}}{r^{2}}-\frac{\Lambda}{3} r^{2}\right) d \varphi^{2}
\end{gathered}
$$


with $C$ a constant, $q_{m}$ a magnetic charge and $B_{(3)}=$ $q_{m} /\left(\sqrt{G} r^{2}\right)$. If we apply the corresponding duality mapping (47), we obtain the electric Melvin-Bonnor analog in the form

$$
\begin{gathered}
d s^{2}=\left(\frac{C}{r}+\frac{q_{e}^{2}}{r^{2}}-\frac{\Lambda}{3} r^{2}\right) d t^{2} \\
-\left(\frac{C}{r}+\frac{q_{e}^{2}}{r^{2}}-\frac{\Lambda}{3} r^{2}\right)^{-1} d r^{2}-r^{2}\left(d \varphi^{2}-d z^{2}\right),
\end{gathered}
$$

and $E_{(1)}=q_{e} /\left(\sqrt{G} r^{2}\right)$.

Notice that in [21] the authors did not consider (53). They only considered the Melvin-Bonnor universe with a purely electric or a purely magnetic field pointing along the $z$ direction, which are connected by a duality rotation. We must note that eq. (53) coincides with the solution (2.3) given in 22]. The magnetic counterpart of the solution (53) in AdS background is given in [23. Here the range of the coordinates dictates the topology of this solution. We can have cylindrical, planar or toroidal topology. For instance, a cylindrical topology has the range $0<r<\infty, 0<\varphi<2 \pi$, and $-\infty<z<\infty$.

It is interesting to note also, that the metric (52) is related to the 4-dimensional AdS soliton 24 with an electric field (in this case $C=0$ and $\Lambda=-3 / l^{2}$ ). The magnetic soliton is connected to the electric one by a duality rotation. The corresponding AdS black hole may be obtained from (52) (with $C=0$ and $\Lambda=-3 / l^{2}$ ) by the duality mapping (49) and after one must analytically continue the obtained metric, $t \longrightarrow i z$ and $z \longrightarrow i t$.

In conclusion, in a static rotationally symmetric $(2+1)$ dimensional gravity only the electrostatic spacetime with a radial electric field is a black-hole solution; the selfconsistent gravitational fields with azimuthal electric or scalar magnetic field are not black-holes. In this case we also can not have a superposition of a radial electric field with an azimuthal electric nor with a magnetic field. Then we have the situation analogous to $(3+1)$ dimensional cylindrical static gravity considered above, where any superposition of the electromagnetic components given by (39) is not suitable. The above situation is induced by the Maxwell tensor (9) (with $B_{()}=: B_{(3)}$ ) in Einstein-Maxwell theory for cylindrically symmetric spacetimes. On the other hand, the magnetic MelvinBonnor universe (as well as the solution with an azimuthal electric field) is not a black hole; while the electric Melvin-Bonnor universe (53) with a radial electric field and $\Lambda=0$ has a cosmological horizon as well as the electrovacuum $(2+1)$-static solution found in [4], which is the same solution (3) for $\Lambda=0$. From (53) we see that if $\Lambda<0$ we have a cylindrical charged black hole or black string [22], for which the BTZ solution (3) is the $(2+1)$-dimensional absolute analog. Furthermore, if $q=0$, we have a black string solution only because we have included a negative cosmological constant and then this spacetime is asymptotically anti-de Sitter as well as occurs in $(2+1)$-dimensions.

I thank Paul Minning and Carol Muñoz for carefully reading and typing this manuscript respectively. I also wish to thank Alberto García for informative comments. This work was supported by CONICYT through Grant FONDECYT N ${ }^{0} 1010485$ and by Dirección de Promoción y Desarrollo de la Universidad del Bío-Bío.
[1] F. Kottler, Annalen Physic 56, 410 (1918).

[2] J.R. Gott, III and M. Alpert, Gen. Relativ. Gravit. 16, 243 (1984).

[3] S. Giddings, J. Abbott and K. Kuchar, Gen. Rel. Gravit. 16, 751 (1984).

[4] J.R. Gott, III, J.Z. Simon and M. Alpert, Gen. Relativ. Gravit. 18, 1019 (1985).

[5] M. Bañados, C. Teitelboim and J. Zanelli, Phys. Rev. Lett. 69, 1849 (1992).

[6] E. Hirschmann and D. Welch, Phys. Rev. D 53, 5579 (1996).

[7] M. Cataldo and P. Salgado, Phys. Rev. D 54, 2971 (1996).

[8] G. Clément, Class. Quantum Grav. 10, L49 (1993).

[9] C. Martínez, C. Teitelboim and J. Zanelli, Phys. Rev. D 61, 104013 (2000).

[10] M. Kamata and T. Koikawa, Phys. Lett. B 353, 196 (1995).

[11] M. Kamata and T. Koikawa, Phys. Lett. B 391, 87 (1997).

[12] M. Cataldo and P. Salgado, Phys. Lett. B 448, 20 (1999).

[13] K.C.K. Chan, Phys. Lett. B 373, 296 (1996).

[14] G. Clément, Phys. Lett 367, 70 (1996).
[15] S. Fernando and F. Mansouri, Comm. Math. Theor. Phys. 1, 14 (1998).

[16] O.J.C. Dias and J.P.S. Lemos, Rotating magnetic solution in three dimensional Einstein gravity, hepth/0201058.

[17] R. Wald, General Relativity, The University Chicago Press (1984), Chapter 6.

[18] S. Chandrasekhar, The mathematical theory of black holes, Clarendon Press Oxford, Oxford University Press New York (1983), Chapter 2.

[19] G. Clément and A. Fabbri, Class. Quantum Grav. 16, 323 (1999); 17, 2537 (2000).

[20] L. Witten (Ed.), Gravitation: an introduction to current research, Wiley, New York, London, 382 (1962).

[21] W.B. Bonnor, Proc. Phys. Soc. Lond. A 67 (1954), 225; M. A. Melvin, Phys. Lett 8, 65 (1964).

[22] J.P.S. Lemos and V.T. Zanchin, Phys. Rev. D 54, 3840 (1996).

[23] O.J.C. Dias and J.P.S. Lemos, Magnetic strings in antide Sitter General Relativity, hep-th/0110202.

[24] G. Horowitz and R.C. Myers, Phys. Rev. D 59, 026005 (1998). 